\title{
Ethics of online screening for mental illnesses: A systematic review
}

\author{
T. Hassem \& S. Laher
}

\begin{abstract}
Introduction
According to the Global Burden of Disease (GBD) study in 2017, for over 3 decades mental disorders accounted for over $14 \%$ of the standardised age (Ds). In South Africa the lifetime prevalence of a mental disorder was 30. 3\% in 2009 (Herman et al., 2009). In a country like South Africa where access to mental health care and resources are limited (Lund, Kleintjes, Kakuma, Flisher, \& the MHaPP Research Programme Consortium, 2010) and internet access is on the increase, it would be sensible to explore opportunities for using online media to promote mental health. In 2017, 61.8\% of households in South Africa had at least one family member who had access to the internet and $56.9 \%$ of South Africans had mobile internet access (Statistical release, 2017). One of the ways that an online medium may be used in this context of decreasing resources is for mental health screening. The possibility for online screening of mental illnesses holds the promise of early detection and intervention. However, this shifts the interaction typically between a face-toface engagement between two individuals to an online engagement that is not contained with an appropriately trained professional. Given this, it is vital that the ethical considerations associated with this new space be explored. Therefore, this study proceeded with a systematic review to explore the literature for guidance in terms of the online screening for mental health
\end{abstract}

\begin{tabular}{|c|c|c|c|}
\hline Search engines & Search terms & Inclusion Criteria & Exclusion Criteria \\
\hline $\begin{array}{l}\cdot \text { PubMed } \\
\text {-PsychInfo } \\
\text {-Sabinet } \\
\text {-EBSCOHost: } \\
\text { Academic Search } \\
\text { Ultimate, } \\
\text { Psychological and } \\
\text { Behavioural } \\
\text { Sciences Collection }\end{array}$ & $\begin{array}{l}\text { •Ethic* OR "Ethical } \\
\text { Principles" OR } \\
\text { "Ethical } \\
\text { considerations" } \\
\text { AND "online" OR } \\
\text { "Internet" OR "Web- } \\
\text { based" AND } \\
\text { "Screening" OR } \\
\text { "Assessment" AND } \\
\text { "Mental Health" OR } \\
\text { "Mental Illness" OR } \\
\text { "Psychopathology" } \\
\text { OR "Psychological" }\end{array}$ & $\begin{array}{l}\cdot \text { Discussion regarding } \\
\text { the ethics of online } \\
\text { screening or } \\
\text { assessments for } \\
\text { screening or } \\
\text { diagnosis } \\
\text {-197-2019 } \\
\text {-English }\end{array}$ & $\begin{array}{l}\text { - No reference to } \\
\text { online testing, } \\
\text { screening or } \\
\text { assessment } \\
\text {-Discussion of } \\
\text { computerised } \\
\text { assessment or } \\
\text { telepsychology } \\
\text { without mention of } \\
\text { online internet tests } \\
\text { or assessment }\end{array}$ \\
\hline
\end{tabular}

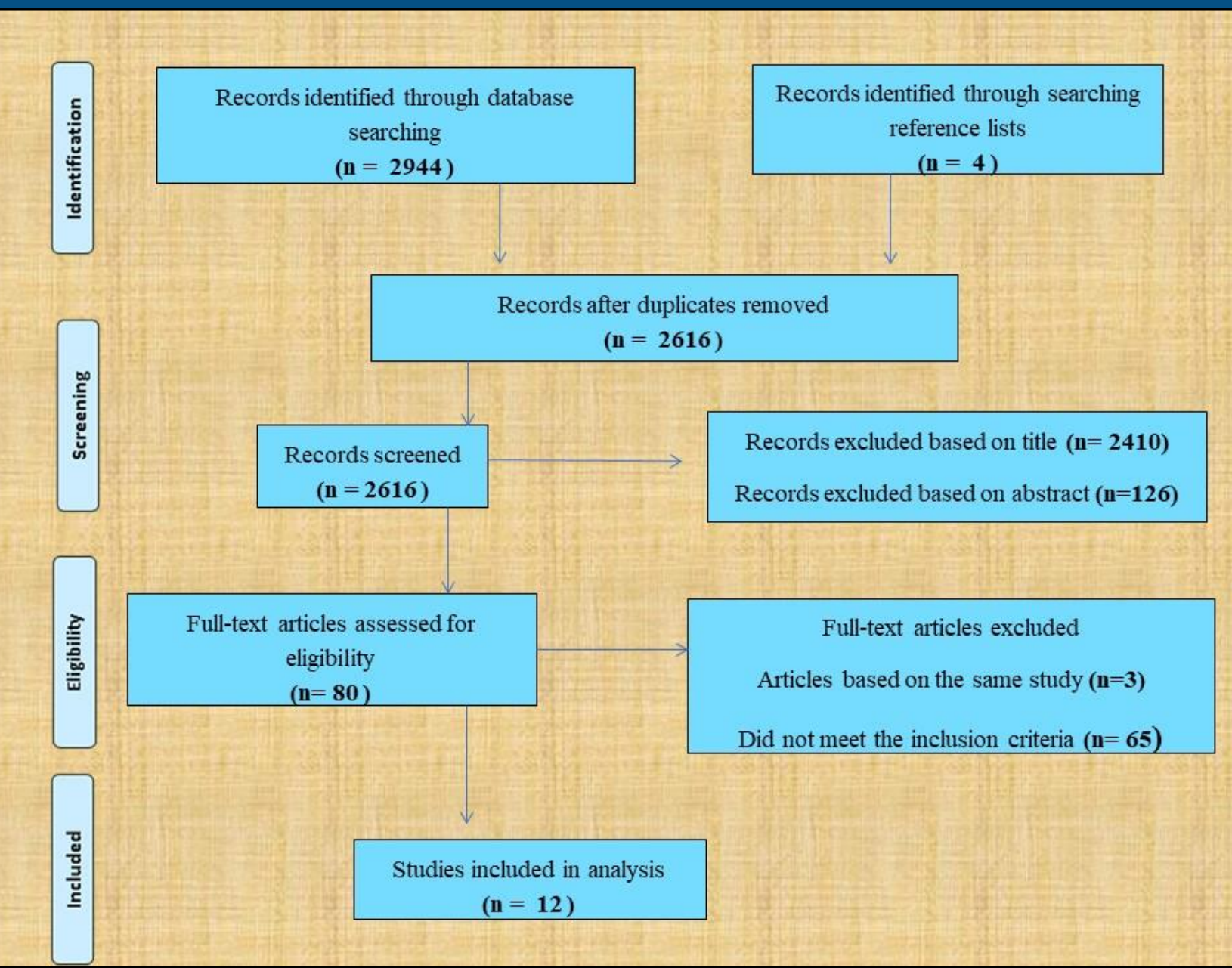

This work is based on the research supported wholly / in part by the National Research Foundation of South Africa (Grant Numbers: 116327 \& 112948)

\section{Review findings}

* 3 articles provided guidelines for internet testing (APS, 2018; Evans, 2018; ITC, 2005)

* 9 articles only highlighted ethical considerations (Barak \& Buchanan, 2004; Bartram, 2006; Buchanan, 2002; Butcher et al., 2004; Fisher \& Fried, 2003, 2008; Joint Task Force, 2013; Kier \& Molinari, 2004; Luxton et al., 2016; Naglieri et al., 2004, 2008)

* All authors suggest that psychological guidelines developed for normal pen-paper tests should be adhered to and highlighted additional guidelines and considerations unique to internet testing.

* Barak and Buchanan (2004), Butcher et al. (2004), Kier and Molinari (2004), Luxton et al. (2016), as well as Naglieri et al. (2004, 2008) highlight that online psychological testing currently does not adhere to the all guidelines for psychological testing and therefore called for the development of formal guidelines specific to internet testing.

* Through a thematic analysis of the twelve included articles, five core themes emerged.

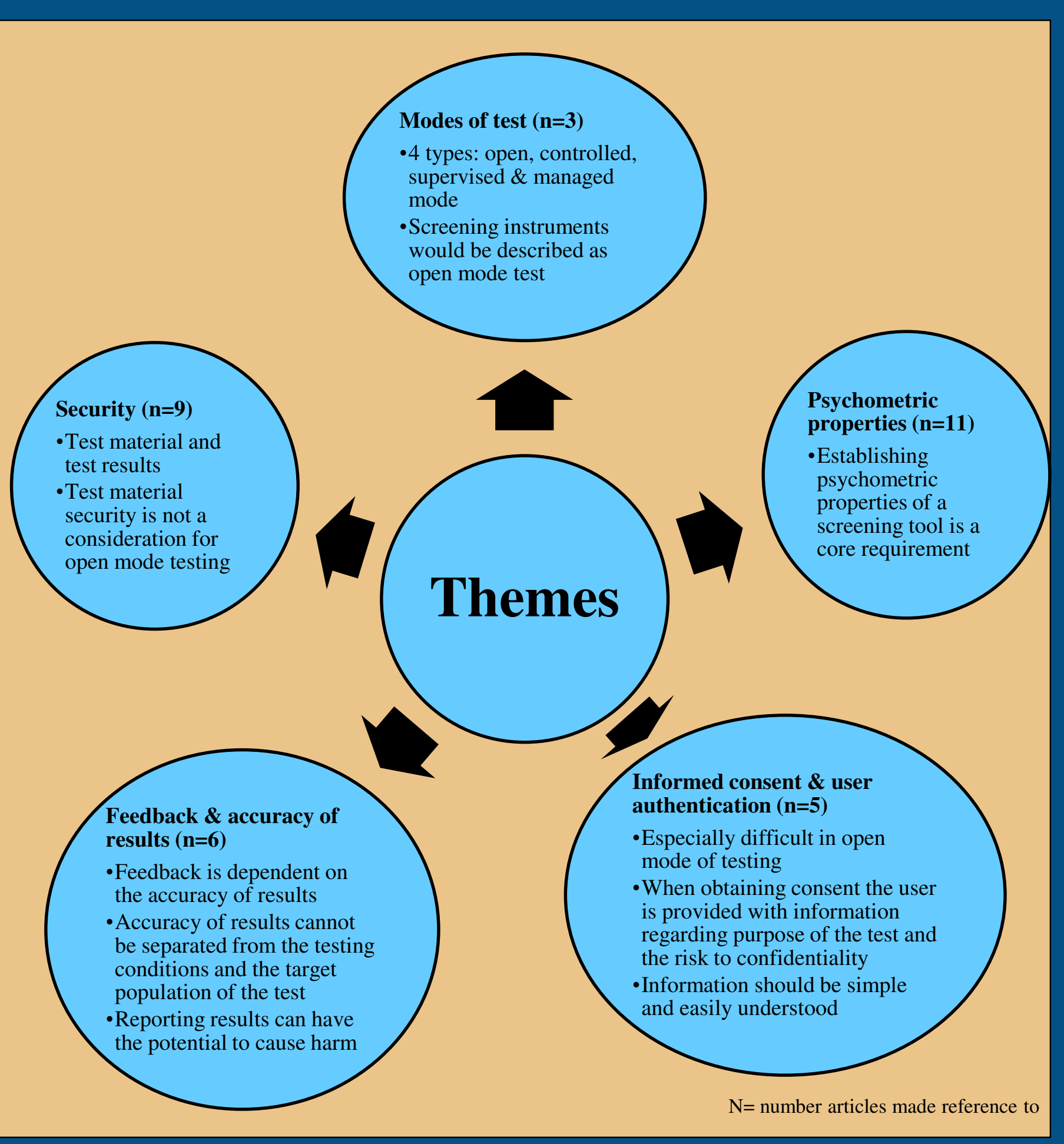

\section{Conclusion}

Results obtained from this study revealed that currently there are no formal and specific guidelines for online mental health screening. The online mental health screening environment allows for exploitation of potentially vulnerable individuals. Therefore, it is necessary given the global accessibility to mental health screening tools, that a formal set of guidelines for online screening to be developed. This review provides clear guidelines for issues to be addressed in ethical guidelines for online screening of mental health and marks the first step towards making online screening of mental health responsible and possible. 